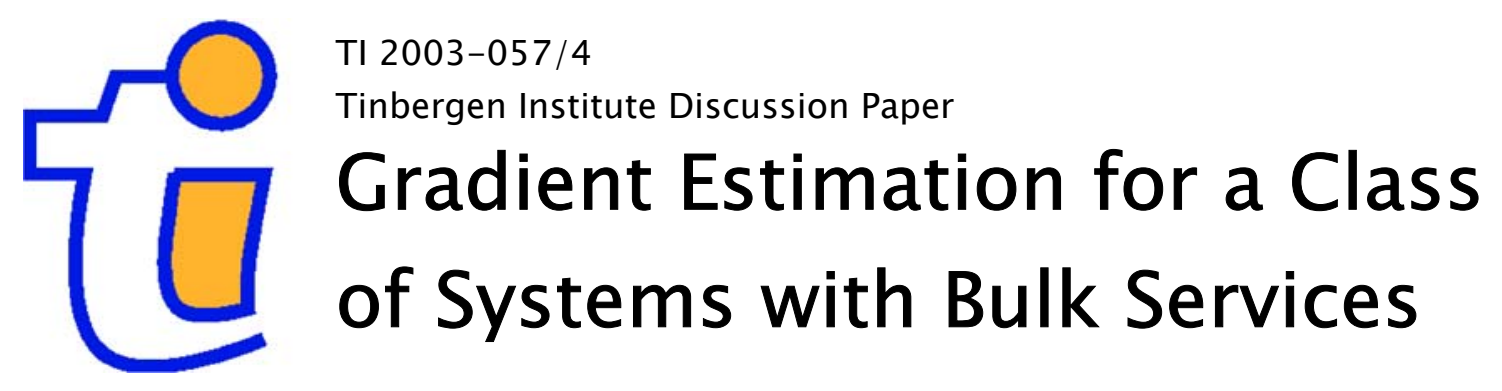

Felisa J. Vázquez-Abad'
Bernd Heidergott ${ }^{2}$

${ }^{\prime}$ Dépt. d'Informatique et Recherche Opérationelle, Université de Montréal, Canada,

2 Faculty of Economics and Business Administration, Vrije Universiteit Amsterdam, and Tinbergen Institute. 


\section{Tinbergen Institute}

The Tinbergen Institute is the institute for economic research of the Erasmus Universiteit Rotterdam, Universiteit van Amsterdam, and Vrije Universiteit Amsterdam.

Tinbergen Institute Amsterdam

Roetersstraat 31

1018 WB Amsterdam

The Netherlands

Tel.: $\quad+31(0) 205513500$

Fax: $\quad+31(0) 205513555$

Tinbergen Institute Rotterdam

Burg. Oudlaan 50

3062 PA Rotterdam

The Netherlands

Tel.: $\quad+31(0) 104088900$

Fax: $\quad+31(0) 104089031$

Please send questions and/or remarks of nonscientific nature to driessen@tinbergen.nl.

Most TI discussion papers can be downloaded at http://www.tinbergen.nl. 


\title{
Gradient estimation for a class of systems with bulk services: a problem in public transportation
}

\author{
Felisa J. Vázquez-Abad * member GERAD \\ Département d'informatique et recherche opérationnelle \\ Université de Montréal, C.P. 6128 Succ Centre-Ville, H3C 3J7 Canada \\ also Principal Investigator, DEEE, University of Melbourne, Australia. \\ and \\ Bernd Heidergott ${ }^{\dagger}$ \\ Vrije Universiteit Amsterdam and Tinbergen Institute \\ De Boelelaan 1105, 1081 HV Amsterdam, the Netherlands, \\ vazquez@iro.umontreal.ca, bheidergott@feweb.vu.nl
}

\begin{abstract}
This paper deals with a system where batch arrivals wait in a station until a server (a train) is available, at which moment it services all customers in waiting. This is an example of a bulk server, which has many applications in public transportation, telecommunications, computer resource allocation, and multiple access telecommuncation networks, among others. We consider a subway model and focus on a metro line serving a particular metro station. Denote the planned inter-departure time of this line by $\theta$. The metro station is served by several other lines and passengers change trains at the station. Traveling times of trains are assumed to be given by fixed times and an additional stochastic noise. We perform a sensitivity analysis of the total delay of passengers waiting for the " $\theta$ " line with respect to $\theta$. We establish a smoothed perturbation analysis (SPA), a measure-valued differentiation (MVD), and a score function (SF) estimator. Numerical experiments are performed to compare the ensuing estimators. It turns out that the SPA and MVD estimators are intrinsically different and the model presented in this paper may serve as a counter-example to the widespread belief that SPA and MVD yield similar estimators.
\end{abstract}

Keywords: sensitivity analysis, smoothed perturbation analysis, score function, measure--valued differentiation, bulk servers.

\footnotetext{
* This work was partly supported by NSERC and FCAR Grants of the Governement of Canada and Québec.

${ }^{\dagger}$ Part of this work was done while the second author was with TU Eindhoven, Dept of Mathematics and Computing Science, Division of Mathematics, the Netherlands
} 


\section{Introduction}

The current paper is a detailed extended version of the results in [12]. The basic model that we will use in this paper is a transportation model suggested by a subway problem. However the methodology introduced to calculate the sensitivies with respect to frequencies is general and can be adapted to other applications. For example in all-optical telecommunication systems, bursts are created at the access networks and are sent to the core network. How to assemble the bursts in an optical burst switched network is still an open problem, but conceivably one could propose to send the bursts by aggregating all the packets that arrive over a given period of time requesting transmission. In such schemes it would be essential to measure the sensitivity to changes in the frequency of burst transmission, making the problem similar to the one under study here. Systems with bulk services arise in a number of important applications. In telecommunications, multiple access protocols (MAP) allocate a common server to a number of sources, possibly with different service requirements. One such scheme is similar to a computer sharing device called a "bus", where the different sources waiting for service are visited in a route. When the server is at a particular station, it serves all the packets or requests in waiting. In flexible manufacturing systems, automated vehicles also take and deliver parts to different stations, yielding a similar model.

The analysis presented in this paper stems from a major research project on the application of (on-line) control techniques to public transportation systems, see $[13,15,14]$. Our model comes directly from [15]. Following the Montreal subway system, the subway network operates without a fixed timetable and only frequencies of trains are given at different day segments. Therefore the expected train inter-departure times at the stations served by this line will be determined by the "headway" at the initial station on the line (say, "trains depart every five minutes"). At several major stations passengers can change trains running on the lines of network. In [15], an analytical formula is obtained for the expected waiting time of passengers that arrive at the given track according to a Poisson process (as is customary to assume for those passengers arriving from outside). Accordingly, in this paper we focus on the waiting time of all other passengers, namely those in transfer from other lines in network. These passengers arrive in bulks, and their inter-arrival times at the transfer track depend on the dynamics of train departures at other lines. For such processes, there is no analytical expression for the expected waiting time.

The main problem that we deal with in this paper is the following. At a particular station, we consider a line the inter-departure times of which are influenced by a parameter, say $\theta$, and we call this line the " $\theta$ " line. The metro station is served by several other lines and passengers change trains at the station. The waiting time of a passenger for the " $\theta$ " line is the time that elapses from the arrival of the passenger at the station until the next arrival of a " $\theta$ " train. The performance indicator, denoted by $L(\theta)$, is the total accumulated waiting time of passengers for the " $\theta$ " line over a fixed period of time, like a day or the morning peak hours. We are interested in estimating the sensitivity of the waiting time of passengers with respect to the headway $\theta$. In order to facilitate the on-line control for the " $\theta$ " line, it is necessary to estimate the sensitivity of the accumulated waiting time with respect to $\theta$. In [13] it was established that the gradient (w.r.t. the headways) of the global perfomance function of 
the whole network can be reconstructed via local derivative estimation, which lends the on-line control problem suitable for decentralised operation. In [15] a decentralised stochastic approximation scheme is presented as a means for adapting the subway frequencies to the changing and unpredictable passenger demands. As shown there, the only derivatives that are required to control the network using stochastic approximation are the local quantities of the form:

$$
\frac{d}{d \theta} \mathrm{E}[L(\theta)]
$$

This sensitivity represents the effect that small changes in the mean interdeparture time of the " $\theta$ " line have on the overall expected accumulated waiting time at the station of interest.

It is well known that if $L(\theta)$ is a.s. Lipschitz continuous in $\theta$ then the IPA estimator (the stochastic derivative $L^{\prime}(\theta)$ ) is unbiased and often yields simple and good derivative estimators. Unfortunately the performance $L(\theta)$ may have discontinuities, as shown in Section 3.1. The main approaches to sensitivity analysis that apply to the proposed problem are: smoothed perturbation analysis (SPA), see [3, 2], the score function method (SF), see [8, 10], and measure-valued differentiation (MVD), see [7, 4]. In order to identify a suitable sensitivity estimator, we derive all three SPA, SF and a MVD estimators for the proposed problem. A series of numerical experiments will be performed to investigate the estimators.

Besides solving the problem on how to estimate the local derivatives required for the decentralised adaptive control of a subway system, the contributions of this paper are also of a theoretical nature. We provide an example where the MVD estimator is not a particular implementation of smoothing, and it differs considerably from the SPA estimator. In addition, we observed a pathological behaviour of the SPA estimator, which we have identified in terms of the estimation conditioned on rare events. The numerical instabilities caused by this can be corrected, as we explain below, albeit at the price of a bias in the estimation. While the SF estimator is easy to implement, we realised that it required many months of observations before accurate estimates are obtained, which can hinder the tracking capabilities of the stochastic approximation operating in real time. It is our development of the MVD code for the parallel computation of the different "phantom" systems that permits computation of the derivatives within reasonable times for adaptation.

The paper is organized as follows. Section 2 introduces the model. Section 3 addresses sensitivity analysis. In particular, the SPA estimator is presented in Section 3.1, the SF estimator is established in Section 3.2 and Section 3.3 is devoted to the MVD estimator. Numerical examples are given in Section 4.

\section{The Metro Model}

Consider the behaviour of the platform occupancy process of the " $\theta$ " line at a particular transfer station, focusing only on the contribution from passengers in transfer from other lines. A model for this process, called the "local" model, was developed and tested in [14]. We summarize here the salient features of the model without proofs. Let $\Theta=[a, b] \subset \mathbb{R}$, with $0<a<b<\infty$, " $\theta$ " trains depart from the platform according to a renewal process $D_{\theta}=\left\{D_{\theta}(t): t \geq 0\right\}$ 
whose inter-jump times are i.i.d. with mean $\theta \in \Theta$. Denote the $j$-th interdeparture time of trains by $Y_{\theta}(j)$. We assume that the first " $\theta$ " train arrives at time $V(0)$ and the $j$-th train arrives at

$$
V_{\theta}(j)=V(0)+\sum_{i=1}^{j} Y_{\theta}(i), \quad j \geq 1 .
$$

The jump epochs of $D_{\theta}$ are thus given by $V(0), V_{\theta}(1), V_{\theta}(2), \ldots$ The initial epoch $V(0)$ is independent of $\theta$ because the first train departure is not affected by the targetted inter-departure time within a particular segment of day. Suppose that a target inter-departure time of five minutes is requested by the controller, $\theta=5$. This control starts effectively at 10:00 AM, but $V(0)$ is the epoch of departure of the last train before 10:00 AM. For simplicity, set the initial time at zero. The results in [14] establish that $Y_{\theta}(j)$ are i.i.d. and approximately distributed according to a Normal distribution with mean $\theta$ and standard deviation $\theta \sigma$, denoted by $\mathcal{N}_{\theta,(\theta \sigma)^{2}}$, for given $\sigma>0$, where we choose $\sigma$ small enough so that the probability of the event $Y_{\theta}(j)<0$ is negligibly small for $\theta \in \Theta$. More precisely, the probability that $Y_{\theta}(j)$ is lower than zero is equal to the probability that a standard normal random variable is lower than $-1 / \sigma$. For example, if $\sigma \leq 0.3$, then the probability that $Y_{\theta}(j)$ is smaller than zero is 0.0005 .

Without loss of generality, fix one of the incoming sources of transfer passengers, that is, a line with a different headway (say $\mu$ ), whose trains stop at the given station. Passengers from this line that transfer to the " $\theta$ " line arrive in groups: at epochs $S(k)$ the $k$-th group of transfer passengers arrives at the track, and the size of the group $k$ is independent of $D_{\theta}(\cdot)$. Denote the total number of arrival epochs of groups of passengers up to time $t$ by $A(t)$ and the interarrival times of groups of passengers by $T(k)$, with $T(k)=S(k)-S(k-1)$, for $k \geq 1$, and $S(0)=0$, where we assume $T(k)$ to constitute an i.i.d. sequence (for details on the model refer to [15]). Finally, departing trains have "infinite" capacity (that is, all passengers waiting in a station can and do enter the next " $\theta$ " train ready for departure). The quantity of interest is the accumulated waiting time of passengers in transfer at the given track in the metro station. The quantity of interest is the accumulated waiting time of passengers in transfer at the given track of the " $\theta$ " line in the subway station. In [15] it is shown that the expected wait of the $k$-th group, given the quantities $S(k), T(k)$ is given by $\rho T(k)\left(V_{\theta}(j)-S(k)\right)$, where $j=D_{\theta}(S(k))+1$ is the index of the train that the group $k$ takes. The constant $\rho$ is the aggregate mean number of transfer passengers per unit time. A surrogate (filtered) expression for the waiting time is therefore:

$$
L(\theta)=\rho \sum_{j=1}^{D_{\theta}(T)} \sum_{k=A\left(V_{\theta}(j-1)\right)+1}^{A\left(V_{\theta}(j)\right)}\left(V_{\theta}(j)-S(k)\right) T(k),
$$

where the the sums vanish if the lower bound is larger than the upper bound.

The platform occupancy process $X_{\theta}(t)$ records the number of passengers waiting at the platform at time $t$, as shown. It is completely described in terms of the model above, and the individual waiting times can be evaluated from the sample paths. 


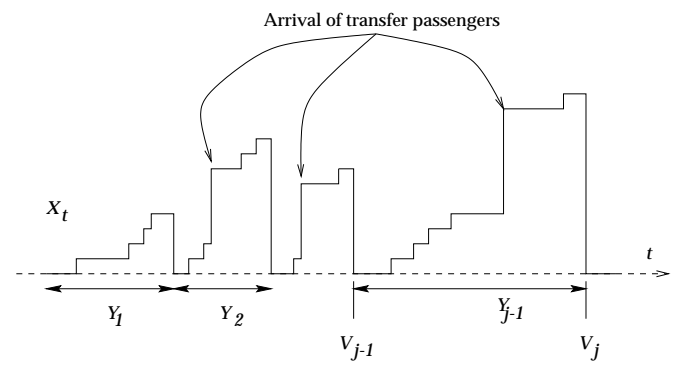

Figure 1: The Platform Occupancy Process

\section{Sensitivity Analysis}

Because $\theta$ is a scaling parameter of $Y_{\theta}(j)$, we can use the representation:

$$
Y_{\theta}(j) \stackrel{\mathcal{L}}{=} \theta \xi(j), \quad V_{\theta}(j) \stackrel{\mathcal{L}}{=} V(0)+\theta \sum_{i=1}^{j} \xi(i),
$$

where $\xi(j)$ is normally distributed with mean 1 and variance $\sigma^{2}$ (and independent of $\theta$ ), and the symbol $\mathcal{L}$ means that the random variables have the same distribution. From (3) follows that $d V_{\theta}(j) / d \theta=\left(V_{\theta}(j)-V(0)\right) / \theta$. If the function $L(\theta)$ were Lipschitz continuous in $\theta$ with probability one, then the stochastic derivative, known as the IPA estimator:

$$
L_{(\mathrm{IPA})}(\theta)=\frac{\rho}{\theta} \sum_{j=1}^{D_{\theta}(T)} \sum_{k=A\left(V_{\theta}(j-1)\right)+1}^{A\left(V_{\theta}(j)\right)}\left(V_{\theta}(j)-V(0)\right) T(k),
$$

would be unbiased [11]. However, $L(\theta)$ fails to be Lipschitz continuous and the discontinuities of this functional are easily described: a negative infinitesimal perturbation of $\theta$ may shift $V_{\theta}(j)$ so that a particular arrival $k^{*}$ may get into the $j$-th " $\theta$ " train for the non-perturbed process and wait for " $\theta$ " $\operatorname{train} j+1$ in the perturbed process. Thus the difference in contribution to the accumulated waiting time will be of order larger than $\mathcal{O}(\Delta \theta)$. It is these discontinuities in the sample performance that prevent the application of IPA. In the presence of discontinuities, the following approaches can be applied in principle: smoothed perturbation analysis (SPA), the score function method (SF) and measure-valued differentiation (MVD). In the following we derive the corresponding different gradient estimators for our model.

\section{$3.1 \quad$ SPA}

This section presents the formulation of an SPA estimator for

$$
\frac{d}{d \theta} \mathrm{E}[L(\theta) \mid V(0), S(0)]
$$

(recall that $V(0)$ is the time of the first departure of a train and $S(0)$ is the first passenger arrival epoch). Write $\mathrm{E}_{0}$ to indicate the conditional expectation given 
the initial values $V(0)$ and $S(0)$. The model for SPA uses common random variables $\{\xi(j)\}$ in (3) for all $\theta$, so that

$$
V_{\theta-\Delta \theta}(j)=\left(1-\frac{\Delta \theta}{\theta}\right) V_{\theta}(j)+\frac{\Delta \theta}{\theta} V(0) .
$$

We say that the $k$-th arrival is a critical event if it satisfies

$$
V_{(\theta-\Delta \theta)}\left(D_{\theta}(S(k))+1\right)<S(k) \leq V_{\theta}\left(D_{\theta}(S(k))+1\right),
$$

in words: if arrival $k$ is a critical event, then transfer passengers arriving in group $k$ get into train $j=D_{\theta}(S(k))+1$ at the given platform for the nominal process at $\theta$, whereas they miss train $j$ in the perturbed process at $\theta-\Delta \theta$ and will have to wait until later, as represented in Figure 2.

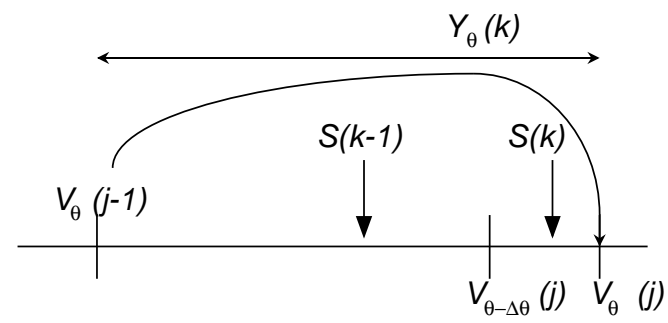

Figure 2: Critical Event

Recall that $\left\{Y_{\theta}(k)\right\}$ are assumed to be i.i.d. normal with density

$$
\phi_{\theta}(x) \triangleq \phi_{\theta,(\theta \sigma)^{2}}(x)=\frac{1}{\sqrt{2 \pi \sigma^{2} \theta^{2}}} e^{-\frac{(x-\theta)^{2}}{2 \theta^{2} \sigma^{2}}}
$$

and denote the cumulative density function (c.d.f.) of $Y_{\theta}(j)$ by $\Phi_{\theta}$.

\section{Theorem 1 If}

$$
\mathrm{E}\left[\sup _{\theta \in \Theta} D_{\theta}(T)\right]<\infty \quad \text { and } \mathrm{E}[A(T)]<\infty,
$$

then the SPA estimator

$$
\begin{aligned}
L_{(\mathrm{SPA})}(\theta)=\rho \sum_{j=1}^{D_{\theta}(T)} & \sum_{k=A\left(V_{\theta}(j-1)\right)+1}^{A\left(V_{\theta}(j)\right)} T(k) \\
& \times\left(\frac{V_{\theta}(j)-V(0)}{\theta}-\frac{(S(k)-V(0)) \phi_{\theta}\left(S(k)-V_{\theta}(j-1)\right)}{1-\Phi_{\theta}\left(S(k)-V_{\theta}(j-1)\right)}\right)
\end{aligned}
$$

is unbiased for the desired derivative, that is,

$$
\mathrm{E}_{0}\left[L_{(\mathrm{SPA})}(\theta)\right]=\frac{d}{d \theta} \mathrm{E}_{0}[L(\theta)] .
$$

Proof: The SPA estimator will be built using the limit of the finite differences:

$$
\mathrm{E}_{0}\left[\frac{L(\theta)-L(\theta-\Delta \theta)}{\Delta \theta}\right], \quad \Delta \theta \neq 0 .
$$


Consider the filtration $\mathfrak{F}(k)=\sigma\left(S(1), \ldots, S(k) ; V_{\theta}(1), \ldots, V_{\theta}\left(D_{\theta}(S(k))\right)\right)$ which contains the history of the process up to arrival epoch $S(k)$. Call $\Omega_{\Delta \theta}(k) \subset$ $\Omega, k=1,2, \ldots$, the event set where the $k$-th arrival is the first critical event of the process, and call $\Omega^{*}(\Delta \theta)=\Omega \backslash \cup_{k} \Omega_{\Delta \theta}(k)$ the set of all trajectories where no discontinuities occur. Then

$$
\begin{aligned}
\mathrm{E}_{0}[ & \left.\frac{L(\theta)-L(\theta-\Delta \theta)}{\Delta \theta}\right] \\
= & \underbrace{\mathrm{E}_{0}\left[\mathrm{E}\left[\frac{L(\theta)-L(\theta-\Delta \theta)}{\Delta \theta} \mid \Omega^{*}(\Delta \theta)\right] \mathrm{P}\left(\Omega^{*}(\Delta \theta)\right)\right]}_{[a]} \\
& +\underbrace{\mathrm{E}_{0}\left[\sum_{k=1}^{A(T)} \mathrm{E}\left[L(\theta)-L(\theta-\Delta) \mid \Omega_{\Delta \theta}(k)\right] \frac{\mathrm{P}\left(\Omega_{\Delta \theta}(k)\right)}{\Delta}\right]}_{[b]} .
\end{aligned}
$$

In order to calculate the limit as $\Delta \theta \rightarrow 0$ for expression [b], we calculate the contribution from each of the critical events, conditioning on the history of the process, that is, the limits of the form:

$$
\lim _{\Delta \theta \rightarrow 0} \mathrm{E}\left[\mathrm{E}\left[L(\theta)-L(\theta-\Delta \theta) \mid \Omega_{\Delta \theta}(k), \mathfrak{F}(k)\right] \frac{\mathrm{P}\left(\Omega_{\Delta \theta}(k) \mid \mathfrak{F}(k)\right)}{\Delta \theta}\right],
$$

for each $k$. The above limit is evaluated in two steps, first calculation of the critical rates:

$$
p_{\theta}(k)=\lim _{\Delta \theta \rightarrow 0} \frac{\mathrm{P}\left(\Omega_{\Delta \theta}(k) \mid \mathfrak{F}(k)\right)}{\Delta \theta},
$$

and then calculation of the limiting value of the difference process: $L(\theta)-L(\theta-$ $\Delta \theta)$, given the critical event.

Critical rates: We now calculate the probability of a critical event given the history up to the $k$-th arrival. The critical event $\Omega_{\Delta \theta}(k)$ conditions on the fact that the $D_{\theta}(S(k))$-th train was the most recent departure. Let $Y_{\theta}^{*}$ be the interdeparture time $Y_{\theta}\left(D_{\theta}(S(k))\right)$ conditioned on the event that the departure of the next train (that is train $D_{\theta}(S(k))+1$ ) takes place after epoch $S(k)$, that is, it has the conditional distribution of $Y_{\theta}$ conditioned on the event that $Y_{\theta}$ is greater than $S(k)-V_{\theta}(j)$, for $j=D_{\theta}(S(k))$. Recall that $\Phi_{\theta}$ denotes the c.d.f. of $Y_{\theta}(k)$. It holds that:

$$
\begin{aligned}
\mathrm{P}\left(\Omega_{\Delta \theta}(k) \mid \mathfrak{F}(k)\right) & \stackrel{(6)}{=} \mathrm{P}\left\{V_{(\theta-\Delta \theta)}\left(D_{\theta}(S(k))+1\right)<S(k) \leq V_{\theta}\left(D_{\theta}(S(k))+1\right) \mid \mathfrak{F}(k)\right\} \\
\stackrel{(5)}{=} & \mathrm{P}\left\{\left(1-\frac{\Delta \theta}{\theta}\right)\left(V_{\theta}(j)+Y_{\theta}^{*}\right)+\frac{\Delta \theta}{\theta} V(0)<S(k) \leq V_{\theta}(j)+Y_{\theta}^{*} \mid \mathfrak{F}(k)\right\} \\
= & \frac{\mathrm{P}\left\{\left(1-\frac{\Delta \theta}{\theta}\right)\left(V_{\theta}(j)+Y_{\theta}\right)+\frac{\Delta \theta}{\theta} V(0)<S(k) \leq V_{\theta}(j)+Y_{\theta} \mid \mathfrak{F}(k)\right\}}{\mathrm{P}\left\{Y_{\theta}>S(k)-V_{\theta}(j)\right\}} .
\end{aligned}
$$

Observe that $S(k) \leq V_{\theta}(j)+Y_{\theta}$ is equivalent to $S(k)-V_{\theta}(j) \leq Y_{\theta}$, and that

$$
\left(1-\frac{\Delta \theta}{\theta}\right)\left(V_{\theta}(j)+Y_{\theta}\right)+\frac{\Delta \theta}{\theta} V(0)<S(k)
$$


is equivalent to

$$
Y_{\theta} \leq\left(\frac{\theta}{\theta-\Delta \theta}\right)\left(S(k)-\frac{\Delta \theta}{\theta} V(0)\right)-V_{\theta}(j) .
$$

Hence,

$$
\begin{aligned}
& \mathrm{P}\left(\Omega_{\Delta \theta}(k) \mid \mathfrak{F}(k)\right) \\
& \quad=\frac{\mathrm{P}\left\{S(k)-V_{\theta}(j)<Y_{\theta} \leq\left(\frac{\theta}{\theta-\Delta \theta}\right)\left(S(k)-\frac{\Delta \theta}{\theta} V(0)\right)-V_{\theta}(j) \mid \mathfrak{F}_{k}\right\}}{1-\Phi_{\theta}\left(S(k)-V_{\theta}(j)\right)} .
\end{aligned}
$$

Use now the Taylor series expansion $\theta /(\theta-\Delta \theta) \approx 1+\Delta \theta / \theta+\mathcal{O}\left(\Delta \theta^{2}\right)$ together with the fact that $Y_{\theta}$ has a bounded density to obtain:

$$
\begin{aligned}
\lim _{\Delta \theta \rightarrow 0} & \frac{\mathrm{P}\left(\Omega_{\Delta \theta}(k) \mid \mathfrak{F}(k)\right)}{\Delta \theta} \\
\quad= & \lim _{\Delta \theta \rightarrow 0} \frac{\Phi_{\theta}\left(S(k)-V_{\theta}(j)\right)-\Phi_{\theta}\left(S(k)-V_{\theta}(j)+(S(k)-V(0)) \frac{\Delta \theta}{\theta}\right)}{\Delta \theta\left[1-\Phi_{\theta}\left(S(k)-V_{\theta}(j)\right)\right]}+\mathcal{O}(\Delta \theta) \\
\quad= & \left(\frac{S(k)-V(0)}{\theta}\right) \frac{\phi_{\theta}\left(S(k)-V_{\theta}(j)\right)}{1-\Phi_{\theta}\left(S(k)-V_{\theta}(j)\right)} .
\end{aligned}
$$

The above result implies that

$$
\lim _{\Delta \theta \rightarrow 0} \mathrm{P}\left(\Omega_{\Delta \theta}(k)\right)=\lim _{\Delta \theta \rightarrow 0} \mathrm{E}\left[\mathrm{P}\left(\Omega_{\Delta \theta}(k) \mid \mathfrak{F}(k)\right)\right]=0,
$$

for a proof use the fact that $S(k) \leq T$ a.s. in (9), together with Dominated Convergence. Using that each term $\mathrm{P}\left(\Omega_{\Delta \theta}(k)\right)$ is uniformly bounded and $\mathrm{E}[A(T)]<$ $\infty$, we obtain

$$
\lim _{\Delta \theta \rightarrow 0} \mathrm{P}\left(\Omega^{*}(\Delta \theta)\right)=1-\mathrm{E}\left[\sum_{k=1}^{A(T)} \lim _{\Delta \theta \rightarrow 0} \mathrm{P}\left(\Omega_{\Delta \theta}(k)\right)\right]=1 .
$$

In addition to that, if $\mathrm{E}\left[\sup _{\theta \in \Theta} D_{\theta}(T)\right]$ is finite, then the term [a] inside the expectation $E_{0}$ in equation (8) converges to the IPA derivative $(4), L_{(\text {IPA })}(\theta)$, that is,

$$
\mathrm{E}_{0}\left[L_{(\mathrm{IPA})}(\theta)\right]=\lim _{\Delta \rightarrow 0}[a]
$$

Difference process: Given $\Omega_{\Delta \theta}(k)$ and $\mathfrak{F}(k)$, all passengers in arrivals $m<k$ take the same train and the contribution to the difference process is

$$
T(m)\left(V_{\theta}\left(D_{\theta}(S(m))+1\right)-S(m)\right)-T(m)\left(V_{(\theta-\Delta \theta)}\left(D_{\theta}(S(m))+1\right)-S(m)\right),
$$

which is of order $\mathcal{O}(\Delta \theta)$, because all passengers in arrivals $m<k$ take the same train, namely $D_{\theta}(S(m))=D_{\theta-\Delta \theta}(S(m))$ and the difference in their wait is infinitesimal. Provided that the perturbation is small enough, the definition of the critical event implies that passengers arriving in train $k$ board train $D_{\theta}(S(k))+1$ in the nominal trajectory have to wait for train $D_{\theta}(S(k))+2$ in the perturbed path. Thus the contribution to $\Delta L$ is

$$
\begin{aligned}
& \rho \mathrm{E}_{0}\left[T(k)\left[V_{\theta}\left(D_{\theta}(S(k))+1\right)-V_{(\theta-\Delta \theta)}\left(D_{\theta}(S(k))+2\right)\right] \mid \Omega_{\Delta \theta}(k), \mathfrak{F}(k)\right] \\
& \quad=-\rho \theta T(k)+\mathcal{O}(\Delta \theta) .
\end{aligned}
$$


For passengers in future trains $k+m, m>0$, the contribution is again infinitesimal, even if $k+m$ happens to be a secondary critical event. By a secondary critical event we mean that given $\Omega_{\Delta \theta}(k)$, the passengers arriving in incoming trains $k+l$ board the same outgoing train in both trajectories, for $l<m$, but those arriving at time $S(k+m)$ just missed train $D_{\theta}(S(k)+1)$ in the perturbed trajectory. While the contribution to the expected wait of those passengers is proportional to $\rho \theta T(k+m)$ (using the same calculation as before), it can be shown that the probability of a secondary critical event given $\Omega_{\Delta \theta}(k)$ and $\mathfrak{F}(k)$ is $\mathcal{O}(\Delta \theta)$. Hence, the contribution of a second critical event (whenever it may occur) is zero. Applying an induction argument it follows that the contribution of any sample path with more than one critical event to the derivative is zero and thus

$$
\lim _{\Delta \theta \rightarrow 0} \mathrm{E}_{0}\left[L(\theta)-L(\theta-\Delta \theta) \mid \Omega_{\Delta \theta}(k), \mathfrak{F}(k)\right]=-\rho \theta T(k) .
$$

Finalizing: From (9) and (11), it now follows that

$$
\begin{aligned}
\lim _{\Delta \theta \rightarrow 0} \mathrm{E}_{0}\left\{\sum_{k=1}^{A(T)} \mathrm{E}_{0}\left[L(\theta)-L(\theta-\Delta \theta) \mid \Omega_{\Delta \theta}(k), \mathfrak{F}(k)\right] \frac{\mathrm{P}\left(\Omega_{\Delta \theta}(k) \mid \mathfrak{F}(k)\right)}{\Delta \theta}\right\} \\
=-\rho \mathrm{E}_{0}\left\{\sum_{k=1}^{A(T)} T(k)(S(k)-V(0)) \frac{\phi_{\theta}\left(S(k)-V_{\theta}\left(D_{\theta}(S(k))\right.\right.}{1-\Phi_{\theta}\left(S(k)-V_{\theta}\left(D_{\theta}(S(k))\right)\right)}\right\}
\end{aligned}
$$

for the SPA contribution of the derivative estimator. Following (8), combining the above SPA contribution with the IPA contribution (10) completes proof.

QED

By Theorem 1, a sufficient condition for unbiasedness of the SPA estimator is $\mathrm{E}_{0}\left[\sup _{\theta \in \Theta} D_{\theta}(T)\right]<\infty$ and $\mathrm{E}_{0}[A(T)]<\infty$, which is satisfied for the metro model.

\subsection{Score Function}

The score function estimator is presented in the following theorem.

\section{Theorem 2 If}

$$
\mathrm{E}\left[\sup _{\theta \in \Theta}\left(D_{\theta}(T)\right)^{2}\right]<\infty
$$

then the score function estimator

$$
L_{(\mathrm{SF})}=\left(\frac{1}{\theta^{3} \sigma^{2}} \sum_{i=1}^{D_{\theta}(T)+1}\left(\left(Y_{\theta}(i)\right)^{2}-\theta Y_{\theta}(i)\right)-\frac{D_{\theta}(T)+1}{\theta}\right) L(\theta)
$$

is unbiased for the derivative (1).

Proof: Within this proof only, we shall adopt the more compact notation $\left(Y_{1}, \ldots, Y_{n}\right)$, dropping the dependency in $\theta$. The proof is performed in two stages. First, we use the fact that for any adapted stopping time $\tau$ the event 
$\{\tau=n\}$ can be determined with the knowledge of the history up to time $n$, i.e. $\left(Y_{1}, \ldots, Y_{n}\right)$. The following change of measure holds:

$$
\mathrm{E}\left[h\left(\tau ; Y_{1}, \ldots, Y_{\tau}\right)\right]=\mathrm{E}\left[L_{\theta}\left(\tilde{\tau} ; \tilde{Y}_{1}, \ldots, \tilde{Y}_{\tau}\right) h\left(\tilde{\tau} ; \tilde{Y}_{1}, \ldots, \tilde{Y}_{\tau}\right)\right]
$$

for any real valued function $h$, where $\tilde{Y}_{i}$ are i.i.d. normal $\mathcal{N}\left(1, \sigma^{2}\right)$ and the Radon-Nikodym derivative $L_{\theta}$ is defined by:

$$
L_{\theta}\left(n ; \tilde{Y}_{1}, \ldots, \tilde{Y}_{n}\right)=\prod_{i=1}^{n} \frac{\phi_{\theta}\left(\tilde{Y}_{i}\right)}{\phi_{1}\left(\tilde{Y}_{i}\right)} .
$$

Notice that the density $\phi_{1}(\cdot)$ is simply the normal density with mean 1 and variance $\sigma^{2}$, see (7). This result follows from the Monotone Convergence Theorem. Indeed, it holds that

$$
\begin{aligned}
& \mathrm{E}\left[h\left(\tau ; Y_{1}, \ldots, Y_{\tau}\right)\right] \\
& =\mathrm{E}\left[\lim _{n \rightarrow \infty} \sum_{i=1}^{n} h\left(\tau ; Y_{1}, \ldots, Y_{n}\right) \mathbf{1}_{\{\tau=n\}}\right] \\
& =\lim _{n \rightarrow \infty} \mathrm{E}\left[\sum_{i=1}^{n} h\left(\tau ; Y_{1}, \ldots, Y_{n}\right) \mathbf{1}_{\{\tau=n\}}\right] \\
& =\lim _{n \rightarrow \infty} \sum_{i=1}^{n} \mathrm{E}\left[h\left(n ; \tilde{Y}_{1}, \ldots, \tilde{Y}_{n}\right) \mathbf{1}_{\{\tilde{\tau}=n\}} L_{\theta}\left(n ; \tilde{Y}_{1}, \ldots, \tilde{Y}_{n}\right)\right] \\
& =\mathrm{E}\left[h\left(\tilde{\tau} ; \tilde{Y}_{1}, \ldots \tilde{Y}_{\tilde{\tau}}\right) L_{\theta}\left(\tilde{\tau} ; \tilde{Y}_{1}, \ldots, \tilde{Y}_{\tilde{\tau})}\right),\right.
\end{aligned}
$$

which establishes (13).

The proof of the theorem is completed by noticing that under the change of measure the stopping time $\tilde{\tau}$ as well as the sample performance $h$ are independent of $\theta$. Therefore, the random variable

$$
h\left(\tilde{\tau} ; \tilde{Y}_{1}, \ldots \tilde{Y}_{\tilde{\tau}}\right) L_{\theta}\left(\tilde{\tau} ; \tilde{Y}_{1}, \ldots, \tilde{Y}_{\tilde{\tau}}\right)
$$

is a.s. Lipschitz continuous in $\theta$, which follows from the assumption that $\theta \in$ $\Theta=[a, b], 0<a<b<\infty$, implying that the normal densities $\phi_{\theta}(\cdot)$ have a uniformly bounded Lipschitz constant. Using Dominated Convergence to interchange derivative and expectation, one obtains:

$$
\begin{aligned}
& \frac{d}{d \theta} \mathrm{E}\left[h\left(\tilde{\tau} ; \tilde{Y}_{1}, \ldots \tilde{Y}_{\tau}\right) L_{\theta}\left(\tilde{\tau} ; \tilde{Y}_{1}, \ldots, \tilde{Y}_{\tilde{\tau}}\right)\right] \\
& =\mathrm{E}\left[h\left(\tilde{\tau} ; \tilde{Y}_{1}, \ldots, \tilde{Y}_{\tilde{\tau}}\right) \frac{d}{d \theta} L_{\theta}\left(\tilde{Y}_{1}, \ldots, \tilde{Y}_{\tilde{\tau}}\right)\right] \\
& \quad=\mathrm{E}\left[h\left(\tilde{\tau} ; \tilde{Y}_{1}, \ldots, \tilde{Y}_{\tilde{\tau}}\right)\left(\frac{d}{d \theta} \prod_{i=1}^{\tilde{\tau}} \phi_{\theta}\left(\tilde{Y}_{i}\right)\right)\left(\prod_{i=1}^{\tilde{\tau}} \phi_{1}\left(\tilde{Y}_{i}\right)\right)^{-1}\right] .
\end{aligned}
$$

Notice that

$$
\frac{d}{d \theta} \prod_{i=1}^{\tilde{\tau}} \phi_{\theta}\left(\tilde{Y}_{i}\right)=\left(\frac{d}{d \theta} \ln \prod_{i=1}^{\tilde{\tau}} \phi_{\theta}\left(\tilde{Y}_{i}\right)\right) \prod_{i=1}^{\tilde{\tau}} \phi_{\theta}\left(\tilde{Y}_{i}\right)
$$


and applying the same kind of change of measure as in the first part of the proof yields

$$
\begin{aligned}
& \mathrm{E}\left[h\left(\tilde{\tau} ; \tilde{Y}_{1}, \ldots, \tilde{Y}_{\tilde{\tau}}\right)\left(\frac{d}{d \theta} \prod_{i=1}^{\tilde{\tau}} \phi_{\theta}\left(\tilde{Y}_{i}\right)\right)\left(\prod_{i=1}^{\tilde{\tau}} \phi_{1}\left(\tilde{Y}_{i}\right)\right)^{-1}\right] \\
& =\mathrm{E}\left[h\left(\tau, Y_{1}, \ldots, Y_{\tau}\right)\left(\frac{d}{d \theta} \ln \prod_{i=1}^{\tau} \phi_{\theta}\left(Y_{i}\right)\right)\right] \\
& =\mathrm{E}\left[h\left(Y_{\theta}(1), \ldots Y_{\theta}(\tau)\right)\left(\frac{1}{\sigma^{2} \theta^{3}} \sum_{i=1}^{\tau}\left[Y_{\theta}(i)^{2}-\theta Y_{\theta}(i)\right]-\frac{\tau}{\theta}\right)\right],
\end{aligned}
$$

where we have used the fact that $\frac{d}{d \theta}\left(\ln \phi_{\theta}(x)\right)=\left(x^{2}-\theta x\right) / \sigma^{2} \theta^{3}-1 / \theta$, which completes the proof of the claim, identifying $\tau=D_{\theta}(T)+1$ (notice that $D_{\theta}(T)$ is not a stopping time because it cannot be determined from the observation of only $\left.\left(Y_{1}, \ldots, Y_{D_{\theta}(T)}\right)\right)$.

\section{QED}

By Theorem 2, a sufficient condition for unbiasedness of the estimator is that

$$
\mathrm{E}_{0}\left[\sup _{\theta \in \Theta}\left(D_{\theta}(T)\right)^{2}\right]<\infty .
$$

For the metro model the passenger arrival process is obtained via a convolution of fluctuations in the travel time of trains and can be modeled as a renewal process, with a renewal time distribution possessing a finite moment generating function. Because the departure process is also a renewal processes with a.s finite second moment, this condition is satisfied.

\subsection{MVD}

We now turn to the MVD estimator. In order to obtain a MVD estimator, the metro model has to be described by a (general state-space) Markov chain. The resulting estimator is then obtained from differentiating the Markov kernel. In the following, we describe the Markov chain model for the metro line and then state the resulting estimator.

Two events are possible: the departure of a " $\theta$ " train and the arrival of (groups of) passengers. Let $\tau_{n}$ denote the time of the $n$-th occurrence of an event, with $\tau_{0}=0$. Denote the residual time until the next arrival of a group of passengers just after $\tau_{n}$ by $R^{A}(n)$ and the residual time until the next departure of a " $\theta$ " train by $R^{D}(n)$. For example, $R^{A}(0)>R^{D}(0)$ indicates that the first event is a departure of a " $\theta$ " train, whereas $R^{A}(0)<R^{D}(0)$ indicates that the first event is constituted by the arrival of passengers. The number of passenger arrivals that have taken place since the last departure of a " $\theta$ " train is denoted by $X_{\theta}(n)$. We describe the system through the Markov chain $Z_{\theta}(n)=\left(X_{\theta}(n), R_{\theta}^{A}(n), R_{\theta}^{D}(n)\right), n \geq 0$, and denote its Markov kernel by $\mathbf{P}_{\theta}$.

Remark: $\left\{X_{\theta}(n)\right\}$ is the embedded jump chain of the platform occupancy process $\left\{X_{\theta}(t)\right\}$ depicted in Figure 1. In particular, $\left\{X_{\theta}(n)\right\}$ is a generalized SemiMarkov chain (and $\left\{X_{\theta}(t)\right\}$ the corresponding generalized Semi-Markov process) and $\left\{Z_{\theta}(n)\right\}$ is the associated Markov chain, see, for example, [16]. 
The state space of $Z_{\theta}(n)$ is $\mathcal{Z}=\mathbb{N} \times[0, T]^{2}$ and we equip $\mathcal{Z}$ with its Borel field. We denote the total number of arrivals of passengers and departures of " $\theta$ " trains in the interval $[0, t]$ by $N_{\theta}(t) \triangleq D_{\theta}(t)+A(t)$, for $t \geq 0$. Note that the time between the $n$-th and $(n+1)$ st event is given by $\min \left(R_{\theta}^{A}(n), R_{\theta}^{D}(n)\right)$. Setting

$$
l\left(x, r^{A}, r^{D}\right)=x \min \left(r^{A}, r^{D}\right), \quad\left(x, r^{A}, r^{D}\right) \in \mathcal{Z},
$$

the accumulated "ersatz" waiting time of (2) reads

$$
\hat{L}(\theta)=\sum_{n=1}^{N_{\theta}(T)} \rho l\left(Z_{\theta}(n)\right),
$$

with $\hat{L}(\theta)=L(\theta)$ a.s.

For $Z_{\theta}(n)=z=\left(x, r^{A}, r^{D}\right) \in \mathcal{Z}$ we distinguish between two cases. If $r^{D}>r^{A}$, then next event will be the arrival of a group of passengers. In this case the state dynamic is independent of $\theta$, which yields

$$
r^{D}>r^{A} \Longrightarrow \frac{d}{d \theta} \mathrm{E}\left[h\left(Z_{\theta}(n+1)\right) \mid Z_{\theta}(n)=z=\left(x, r^{A}, r^{D}\right)\right]=0
$$

for any measurable bounded function $h$ from $\mathcal{Z}$ to $\mathbb{R}$. On the other hand, if $r^{A} \leq r^{D}$, then the transition of $Z_{n}$ depends on $\theta$. Recall that the density of the inter-departure time of " $\theta$ trains is denoted by $\phi_{\theta}:=\phi_{\theta,(\theta \sigma)^{2}}$, see (7) for a formal definition. Straightforward calculation yields

$$
\begin{aligned}
& \frac{d}{d \theta} \mathrm{E}\left[h\left(Z_{\theta}(n+1)\right) \mid Z_{\theta}(n)=z\right] \\
& \quad=\frac{d}{d \theta} \int_{0}^{\infty} \mathrm{E}\left[h\left(Z_{\theta}(n+1)\right) \mid Z_{\theta}(n)=z, R_{\theta}^{D}(n+1)=y\right] \phi_{\theta}(y) d y \\
& \quad=\int_{0}^{\infty} \mathrm{E}\left[h\left(Z_{\theta}(n+1)\right) \mid Z_{\theta}(n)=z, R_{\theta}^{D}(n+1)=y\right] \frac{d}{d \theta} \phi_{\theta}(y) d y .
\end{aligned}
$$

As shown in the Appendix, the derivative of $\phi_{\theta}$ can be written as scaled difference between two densities, denoted by $\phi_{\theta}^{+}$and $\phi_{\theta}^{-}$, respectively, in formula:

$$
\frac{d}{d \theta} \phi_{\theta}(x)=c_{\theta}\left(\phi_{\theta}^{+}-\phi_{\theta}^{-}\right)
$$

where

$$
c_{\theta}=\frac{1+\sigma \sqrt{2 \pi}}{\theta \sigma \sqrt{2 \pi}},
$$

see the Appendix for the definition of $\phi_{\theta}^{+}$and $\phi_{\theta}^{-}$. Inserting (14) into the above expression for the derivative of $\mathrm{E}\left[h\left(Z_{\theta}(n+1)\right) \mid Z_{\theta}(n)=z\right]$ yields

$$
\begin{aligned}
& \frac{d}{d \theta} \mathrm{E}\left[h\left(Z_{\theta}(n+1)\right) \mid Z_{\theta}(n)=z=\left(x, r^{A}, r^{D}\right)\right] \\
& =\mathbf{1}_{\left\{r^{D} \leq r^{A}\right\}} c_{\theta}\left(\int_{0}^{\infty} \mathrm{E}\left[h\left(Z_{\theta}(n+1)\right) \mid Z_{\theta}(n)=z, R_{\theta}^{D}(n+1)=y\right] \phi_{\theta}^{+}(y) d y\right. \\
& \left.\quad-\int_{0}^{\infty} \mathrm{E}\left[h\left(Z_{\theta}(n+1)\right) \mid Z_{\theta}(n)=z, R_{\theta}^{D}(n+1)=y\right] \phi_{\theta}^{-}(y) d y\right) .
\end{aligned}
$$


In words, when the transition of $Z_{\theta}(n)$ is triggered by the departure of a train, the derivative of $\mathrm{E}\left[h\left(Z_{n+1}\right) \mid Z_{n}=z, R_{\theta}^{D}(n+1)=y\right]$ with respect to $\theta$ is obtained as the difference of two scenarios. For the "+" scenario, the new inter-departure time of the train is, with probability

$$
p^{\mu}=\frac{1}{1+\sigma \sqrt{2 \pi}},
$$

equal to the constant $\theta$ plus an additional stochastic noise that follows a Weibull$\left(2,\left(0.5(\theta \sigma)^{-2}\right)\right.$-distribution and, with probability $1-p^{\mu}$ the new inter-departure time is governed by a Maxwell- $\left(\theta,(\theta \sigma)^{2}\right)$-distribution. For the "-" scenario, the new inter-departure time equals, with probability $p^{\mu}$, the constant $\theta$ minus an additional stochastic noise that follows a Weibull- $\left(2,0.5(\theta \sigma)^{-2}\right)$-distribution and, with probability $1-p^{\mu}$ the new travel time is governed by the Normal$\left(\theta,(\theta \sigma)^{2}\right)$-distribution.

Let $\mathbf{P}_{\theta}^{+}$denote the transition kernel describing the "+" scenario and let $\mathbf{P}_{\theta}^{-}$ describe the "-" scenario. Recall that he transition dynamic is independent of $\theta$ whenever the transition is triggered by the arrival of a (group of) passengers; more formally, for $z=\left(x, r^{A}, r^{D}\right) \in \mathcal{Z}$, it holds that

$$
r^{A}<r^{D} \Longrightarrow \mathbf{P}_{\theta}^{+}(\cdot ; z)=\mathbf{P}_{\theta}^{-}(\cdot ; z)=\mathbf{P}_{\theta}(\cdot ; z) \text {. }
$$

With this notation, we can state our above result in concise way by writing

$$
\frac{d}{d \theta} \mathbf{P}_{\theta}=c_{\theta}\left(\mathbf{P}_{\theta}^{+}-\mathbf{P}_{\theta}^{-}\right) .
$$

Such a representation is called a measure-valued derivative in [4]. More precisely, the above line of argument is independent of the particular performance function $h$ and thus holds for any bounded measurable mapping, and to indicate the range of performance functions the above derivative representation applies to, we call it a $L^{1}(\mathcal{Z})$-derivative, where $L^{1}(\mathcal{Z})$ denote the set of bounded measurable mappings from $\mathcal{Z}$ to $\mathbb{R}$.

For $n \geq 1$, let $Z_{\theta}^{+}(n ; j)$ denote a Markov chain with transition kernel $\mathbf{P}_{\theta}$ expect for the transition from the $(j-1)$ st to the $j$-th state. For this particular transition, the kernel is $\mathbf{P}_{\theta}^{+}$. In the same vein, let $Z_{\theta}^{-}(n ; j)$ denote a Markov chain with transition kernel $\mathbf{P}_{\theta}$ expect for the transition from the $(j-1)$ st to the $j$-th state and for this particular transition let the kernel be equal to $\mathbf{P}_{\theta}^{-}$. For the Markov chain $Z_{\theta}^{+}(n ; p)$ (resp. $Z_{\theta}^{-}(n ; p)$ ) we observe $D_{\theta}^{+}(T ; p)$ (resp. $\left.D_{\theta}^{-}(T ; p)\right)$ departures of " $\theta$ " trains in $[0, T]$ and we denote their departure times by $V_{\theta}^{+}(j ; p)$ (resp. $\left.V_{\theta}^{-}(j ; p)\right)$. We define the MVD estimator as follows

$$
\begin{aligned}
L_{\text {(MVD })}(\theta) & =\rho c_{\theta} \sum_{p=1}^{D_{\theta}(T)}\left(\sum_{j=1}^{D_{\theta}^{+}(T ; p)} \sum_{k=A\left(V_{\theta}^{+}(j-1 ; p)\right)+1}^{A\left(V_{\theta}^{+}(j ; p)\right)}\left(V_{\theta}^{+}(j ; p)-S(k)\right) T(k)\right. \\
& \left.-\sum_{n=1}^{D_{\theta}^{-}(T ; j)} \sum_{k=A\left(V_{\theta}^{-}(j-1 ; p)\right)+1}^{A\left(V_{\theta}^{-}(j ; p)\right)}\left(V_{\theta}^{-}(j ; p)-S(k)\right) T(k)\right) .
\end{aligned}
$$

In words, $L_{(\mathrm{MVD})}(\theta)$ is the difference between the performance of the "+" version and that of the "-" version re-scaled by $(1+\sigma \sqrt{2 \pi}) /(\theta \sigma \sqrt{2 \pi})$. 
Theorem 3 If

$$
\mathrm{E}\left[\left(\sup _{\theta \in \Theta} N_{\theta}(2 T)\right)^{3}\right]<\infty
$$

then

$$
\mathrm{E}_{0}\left[L_{(\mathrm{MVD})}(\theta)\right]=\frac{d}{d \theta} \mathrm{E}_{0}[L(\theta)] .
$$

Proof: We give a sketch of the proof. The key ingredient of the proof is to show that $\mathrm{E}_{0}\left[L_{(\mathrm{MVD})}(\theta)\right]$ is finite. To see this, observe that the "+" and "-" versions are obtained by changing one inter-departure time. The most impact this can have on the number of events is that it reduces an inter-departure time to one that is very small $(\approx 0)$. However, this will cause a shift of at most $T$ time units to the left in the departure process of " $\theta$ " trains and thus the number of departures for the perturbed versions is bounded by $D_{\theta}(2 T)$ and it holds that $D_{\theta}(2 T) \leq N_{\theta}(2 T)$. Furthermore, the total number of arrivals of passengers between departures of " $\theta$ " is bounded from above by $N_{\theta}(T)$. It holds that $N_{\theta}(T)<N_{\theta}(2 T)$ and, using standard results from $[4,5]$, it follows that the intergrability of $\left(\sup _{\theta \in \Theta} N_{\theta}(2 T)\right)^{3}$ implies finiteness of $\mathrm{E}_{0}\left[L_{(\mathrm{MVD})}(\theta)\right]$ (and thus unbiasedness of the estimator).

By Theorem 3, a sufficient condition for unbiasedness of the estimator is that $\mathrm{E}_{0}\left[\sup _{\theta \in \Theta}\left(N_{\theta}(2 T)\right)^{3}\right]<\infty$. This condition is the same as in Theorem 2 and for reasons put forward in the preceding section the condition is satisfied for the metro model.

\section{Numerical Examples}

In this section we present the numerical results obtained for simulations with $D=15$ trains. In all our numerical examples below, $\theta=4.0$ and $\sigma=0.1$. The passenger bulk arrivals to the " $\theta$ " line originate from another line in the metro system (say the " $\mu$ "-line) and transfer at the particular station under study towards the chosen platform. Let $q$ represent the number of stations from the one under study where the actual station is located along the " $\mu$ " line. The $k$-th inter-arrival $T(k)$ at the chosen track under study is therefore obtained from the time between the arrival (at the given transfer station) of " $\mu$ "-trains $k-1$ and $k$. Let $\left\{\delta_{l}(k)\right\}$ be sequence of zero mean random variables. We model by $\mu \delta_{0}$ a zero-mean perturbation in the first station where the $k$-th " $\mu$ "-train starts its sojourn, and the fluctuations $\mu \delta_{i}(k)$ represent small perturbations in travel and passenger boarding and walking times of the $k$-th " $\mu$ "-train at the $i$-th track along its path (which is of length $q$ ). For details please refer to [15].

In all our numerical examples below, $\mu=7.3, q=4$ and $\left\{\delta_{l}(k)\right\}$ are i.i.d. uniformly distributed on $(-0.1,0.1)$. The passenger bulk arrival process is characterized by the inter-arrival times generated using $T(k)=\mu+\mu \Delta(k)$, where:

$$
\Delta(k)=\delta_{0}(k)+\sum_{i=1}^{9}\left(\delta_{i}(k)-\delta_{i}(k-1)\right) .
$$


An experiment with finite differences (which required $10^{7}$ simulated days) gave an estimate of

$$
\mathrm{E}[L(\theta)]=111.53 \pm 0.016
$$

and

$$
\frac{d}{d \theta} \mathrm{E}[L(\theta)]=54.59 \pm 2.23
$$

We will use these values as a benchmark for comparison ${ }^{1}$.

\subsection{The SPA Estimator}

The SPA estimator $L_{(\mathrm{SPA})}$ of Section 3.1contains the term:

$$
\frac{\phi_{\theta}\left(S(k)-V_{\theta}(j-1)\right)}{1-\Phi_{\theta}\left(S(k)-V_{\theta}(j-1)\right)},
$$

which is the hazard rate function evaluated at the point $S(k)-V_{\theta}(j-1)$ : the elapsed time between departure of a train and arrival of a passenger group. If $1-\Phi_{\theta}(x)$ is small, then the above fraction can cause a numerical instability in the SPA estimator. Hence, if the critical event is a rare event, then the SPA estimator runs into numerical difficulties. For distributions with decreasing hazard rate, however, if $1-\Phi_{\theta}(x) \approx 0$ then $\phi_{\theta}(x) /\left(1-\Phi_{\theta}(x)\right) \approx 0$ and we therefore may replace the critical rate by

$$
1_{\left\{1-\Phi_{\theta}\left(S(k)-V_{\theta}(j-1)\right)>\epsilon\right\}} \frac{\phi_{\theta}\left(S(k)-V_{\theta}(j-1)\right)}{1-\Phi_{\theta}\left(S(k)-V_{\theta}(j-1)\right)},
$$

where $\epsilon>0$ becomes a design parameter of the estimator. Denote the resulting SPA estimator by $L_{(\mathrm{SPA})_{\epsilon}}(\theta)$ and notice that $L_{(\mathrm{SPA})_{\epsilon}}(\theta)$ is biased. We performed a series of experiments to illustrate the effect of the choice of $\epsilon$ on the estimator. The results in Table 1 were all obtained with $N=10^{4}$ simulated days.

\begin{tabular}{c|c}
$\epsilon$ & $L_{(\mathrm{SPA})_{\epsilon}}$ \\
\hline 0.001 & $2.293 \pm 10.56$ \\
0.05 & $25.251 \pm 9.82$ \\
0.08 & $39.223 \pm 9.50$ \\
0.01 & $5.645 \pm 10.39$ \\
0.1 & $51.079 \pm 9.20$ \\
0.12 & $60.935 \pm 8.91$ \\
0.15 & $72.509 \pm 8.68$
\end{tabular}

Table 1: SPA Etimation

\subsection{The SF Estimator}

The SF estimator $L_{(\mathrm{SF})}$ can be implemented straightforwardly and no numerical instabilities arise. Table 2 shows the results of several experiments with different sample sizes in terms of the simulated days $N$.

\footnotetext{
${ }^{1}$ For $\sigma=0.1$ the probability of $Y_{\theta}(k) \leq 0$ equals $0.7 \times 10^{-23}$.
} 


\begin{tabular}{c|c}
$\mathrm{N}$ & $L_{(\mathrm{SF})}$ \\
\hline 10000 & $47.879 \pm 22.0028$ \\
50000 & $61.347 \pm 9.8675$ \\
100000 & $55.425 \pm 6.9826$
\end{tabular}

Table 2: SF Estimation

\subsection{The MVD Estimator}

The construction of $L_{(\mathrm{MVD})}$ in Section 3.3 requires generation of two phantom processes $\left(V^{ \pm}(j ; p), j=1, \ldots, D^{ \pm}(T ; p)\right)$, for each term $p$ in the sum. There are several ways to implement the MVD estimator, according to the choice of random variables with the required distributions. In the present work we have used common random numbers (crn) for the passenger arrival sequence $S(k)$ for all the phantom processes. The plus and minus processes are calculated using the same sequence of (observed) passenger arrivals $S(k)$ and the train departure processes defined by:

$$
V_{\theta}^{ \pm}(j ; p)= \begin{cases}V_{\theta}(j) & j<p \\ V_{\theta}(p-1)+Y^{ \pm}(p) & j=p \\ V_{\theta}(p-1)+Y^{ \pm}(p)+\sum_{i=p+1}^{j} Y_{\theta}(i) & j>p\end{cases}
$$

where the phantom inter-departure variables $Y^{ \pm}(p)$ are calculated as follows. Let $\left(U_{p} ; p=1, \ldots, D_{\theta}(T)\right)$ be a sequence of i.i.d. uniform variates, $\left(Z_{\theta}(p) ; p=\right.$ $\left.1, \ldots, D_{\theta}(T)\right)$ a sequence of i.i.d. Weibull $\left(2,0.5(\theta \sigma)^{-2}\right)$, and $\left(M_{\theta}(p) ; p=1, \ldots, D_{\theta}(T)\right)$ a sequence of i.i.d. double Maxwell with parameters $\left(\theta,(\theta \sigma)^{2}\right)$ ( refer to the Appendix for the algorithms necessary for the generation of these random variables). These sequences are also mutually independent. When a train $p$ is scheduled for departure in the main simulation, the code generates $Y_{\theta}(p) \stackrel{\mathcal{L}}{=} \mathcal{N}\left(\theta,(\theta \sigma)^{2}\right)$, and the $p$-th phantom processes are started by setting:

$$
\begin{aligned}
& Y^{+}(p)=\left\{\begin{array}{ll}
\theta+Z_{\theta}(p) & U_{p} \leq p^{\mu} \\
M_{\theta}(p) & \text { otherwise }
\end{array},\right. \\
& Y^{-}(p)=\left\{\begin{array}{ll}
\theta-Z_{\theta}(p) & U_{p} \leq p^{\mu} \\
Y_{\theta}(p) & \text { otherwise }
\end{array} .\right.
\end{aligned}
$$

Remark: For our model we have chosen the distribution of the inter-departure times such that the probability of observing a negative inter-departure time is negligible. The MVD estimator however introduces new distributions for the inter-departure times. For example, for the "-" scenario, the inter-departure time may be equal to $\theta-Z_{\theta}(p)$, where $Z_{\theta}(p)$ follows a Weibull $\mathbf{W}_{\left(2,0.5(\theta \sigma)^{-2}\right)}$ distribution. Hence, $P\left(\theta-Z_{\theta}(p) \leq 0\right)=\mathbf{W}_{\left(2,0.5(\theta \sigma)^{-2}\right)}(\theta)$. For the numerical values in our example, we obtain $P\left(4.0-Y^{-}(p) \leq 0\right)=\mathbf{W}_{(2,3.125)}(4.0) \leq$ $2.0 \times 10^{-21}$. For the Maxwell variable we obtain $P\left(M_{\theta}(p) \leq 0\right) \leq 1.0 \times 10^{-20}$.

\subsection{Randomized MVD}

As explained in the Appendix, sampling from a Maxwell distribution can be as much as four times as expensive as sampling from the Weibull distribution. 
A biased version of the MVD estimator uses only the Weibull and can be justified under our assumption that $\sigma$ is relatively small. Indeed, the weight of the sensitivity with respect to the mean is dominant, i.e. $p^{\mu} \approx 1$, and the sensitivity with respect to the standard deviation can be neglected. Disregarding the contribution of the partial derivative with respect to the standard deviation simplifies the implementation of the estimator and decreases the variance. Indeed if $\sigma \approx 0, p^{\mu} \approx 1$ and most of the time only the Weibull scenario will be chosen for the phantom trains. In our case $\sigma=0.1$ (and $p^{\mu}=0.79957$ ).

The formula for the estimator $L_{(\mathrm{MVD})}$ requires to add up $D$ terms (conditioned on having $D$ departures), each corresponding to a difference process where one inter-departure time has been phantomized, creating the plus and minus phantom processes. One implementation of the method would store all the arrival epochs $S(k)$, as well as the train departure times $V_{\theta}(j)$ for the whole day. Next, for each $p=1, \ldots, D$ a simulation is executed reading all the relevant quantities from memory and generating only the required phantom values $Y^{ \pm}(p)$. While this is straightforward, when simulating the dynamics of 15 trains as we did, the program required excessive CPU time because 30 similar scenarios were recreated in series for each simulated day.

To verify the behaviour of the estimation and its approximation using $p^{\mu}=1$, we used the so-called Randomized MVD [1]. Instead of adding up all the contributions, the method first chooses one index $p$ uniformly amongst the $D$ possible trains. Only the term for this random index is evaluated, and the result is multiplied by $D$. This is a common implementation when dealing with MVD estimators, and its validity stems from the fact that, $\mathrm{E}\left[\sum_{j=1}^{D} W(j)\right]=D \mathrm{E}[W(p)]$, where $p$ is a random index chosen uniformly in $\{1, \ldots, D\}$ and $\{W(k)\}$ any sequence of integrable random variables. Table 3 shows the results of the estimation using the randomized version of the MVD estimation method.

\begin{tabular}{c|c||c} 
& Weibull & Weibull / Maxwell \\
\hline$N$ & $L_{(\mathrm{MVD})}$ & $L_{(\mathrm{MVD})}$ \\
\hline 5000 & $55.50 \pm 10.34$ & $49.51 \pm 12.88$ \\
10000 & $59.89 \pm 7.31$ & $52.37 \pm 8.98$ \\
50000 & $58.26 \pm 3.28$ & $54.26 \pm 4.03$
\end{tabular}

Table 3: Randomized MVD Estimation

\subsection{Averaged MVD}

The MVD estimator is built by calculating the difference between the waiting times (or any other performance of interest) in the "+" and the "-" versions of the process, which differ only in one train inter-departure time. Although seemingly two simulations for each term $p$ in $L_{(\mathrm{MVD})}$ are required in parallel, the phantom methodology can be used to integrate the difference process and calculate the estimator directly using only one sample path, without the need for storing information or performing off-line simulations. We now describe in more detail the algorithm.

Fix an index $p$ and consider the plus and minus $p$-th departure processes $V_{\theta}^{ \pm}(j ; p)$. For ease of notation, we will drop the dependency on $\theta$ as well as the explicit dependency on the chosen index $p$, whenever it is redundant. Each pas- 
senger group $k=1, \ldots, A(T)$ will contribute to the difference of the processes an amount:

$$
\Delta W_{k}(p)=\rho T_{k}\left(V^{+}\left(\kappa_{k}^{+}(p) ; p\right)-V^{-}\left(\kappa_{k}^{-}(p) ; p\right) \mathbf{1}_{\left\{p>D_{\theta}\left(S_{k}\right)\right\}},\right.
$$

where $\kappa_{k}^{ \pm}(p)$ is the index of the train in the plus (or minus) $p$-process that passenger group $k$ takes, that is:

$$
\kappa_{k}^{ \pm}(p)=\min \left(j \geq p: V^{ \pm}(k ; p) \geq S_{k}\right) .
$$

The indicator $\mathbf{1}_{\left\{p>D_{\theta}\left(S_{k}\right)\right\}}$ considers only the arrivals after the perturbation occurs. For each train $p=1, \ldots, D_{\theta}(T)$ at most two random variables are generated to define $\Delta V^{ \pm}(p)=V_{p}-V_{p}^{ \pm}(p)$, and notice that $\Delta V^{ \pm}(p)$ can be either positive or negative, regardless of whether it is the "+" or the "-" $p$ process.

In our simulations, once we have evaluated the process up to departure of train $V_{\theta}(j)$, we generate first $Y_{\theta}(j+1)$, and next we generate all the arrivals within the period $\left(V_{\theta}(j), V_{\theta}(j=1)\right.$, that is: $S(k)$, for $A\left(V_{\theta}(j-1)<\right.$ $k \leq A\left(V_{\theta}(j)\right)$. Call $\mathfrak{F}_{j}$ the history generated by all the available information: $\left(V_{\theta}(1), \ldots, V_{\theta}(j) ; S(k) ; k \leq A\left(V_{\theta}(j)\right)\right.$. The goal is to write the MVD estimator in terms of an adapted process, which means that we can write the code to establish a pathwise estimator that only needs to simulate (or observe) the nominal process to calculate the derivative. To do so, we introduce the concept of stochastic domination for this model. Because of the use of crn's, the initial shift $\Delta^{ \pm}(p)=V^{ \pm}(p ; p)-V_{\theta}(p)$ will carry over all other future departures: $\Delta^{ \pm}(p)=V^{ \pm}(j ; p)-V_{\theta}(j) ; j \geq p$.

Definition 1 A phantom process $\left\{V^{ \pm}(j ; p)\right\}$ is a dominating process if $\Delta^{ \pm}(p)>$ 0 (equivalently if $Y^{ \pm}(p)>Y_{\theta}(p)$ ). Otherwise it is called a dominated process.

For all the dominating processes the corresponding index $\kappa_{k}^{ \pm}(p)$ can be easily computed given $\mathfrak{F}_{j}$, for al passenger groups that take train $j$ in the nominal process, that is, $V_{\theta}(j-1) \leq S(k)<V_{\theta}(j)$. Indeed for a dominating process $\Delta^{ \pm}(p)>0$ so that for all these passengers, $\kappa_{k}^{ \pm}(p) \leq j$ w.p.1, as depicted in Figure 3 .

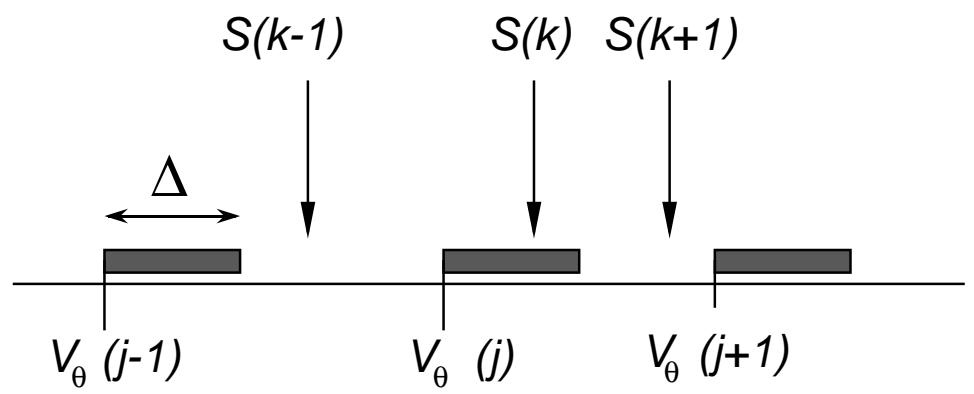

Figure 3: Dominating process

To search for the appropriate index requires simply to keep the index $\kappa_{k}(p)$, and perform the following pseudo-code (we have omitted the label of whether it is a plus or a minus process): 
1. $\kappa_{k-1}[p]$ is known, $p \leq j$

2. Generate $V_{\theta}(j+1)$

3. At arrival epoch $S(k)$ find the index of the phantom trains:

4. For all $p \leq j$ (all phantoms created so far):

$$
\begin{aligned}
& \quad k=\kappa_{k-1}[p], \\
& \text { WHILE }\left(V_{\theta}(k)+\Delta[p]<S(k)\right) \text { DO } \\
& \quad k:=k+1 \\
& \kappa_{k}[p]=k
\end{aligned}
$$

- Add contribution to difference process

5. Initialise $\kappa_{k}[j+1]=j+1$

The contribution of all the phantom processes that affect each of these passenger groups, that is, in the interval $\left[V_{j-1}, V_{j}\right)$ we add the contribution:

$$
\sum_{p=1}^{j} \sum_{k=A\left(V_{\theta}(j-1)\right)+1}^{A\left(V_{\theta}(j)\right)} T_{k}\left( \pm V^{ \pm}\left(\kappa_{k}^{ \pm}(p) ; p\right)\right) \mathbf{1}_{\left\{\Delta^{ \pm}(p)>0\right\}} .
$$

For a dominated process a bit more bookkeeping is necessary in the program, because knowing $\mathfrak{F}_{j}$ is not enough to know what the contribution will be regarding any passenger group $S(k) \in\left(V_{\theta}(j-1), V_{\theta}(j)\right]$ because it may be that $S(k)>V^{ \pm}(j ; p)=V_{\theta}(j)+\Delta^{ \pm}(p)$. To evaluate $\kappa_{k}^{ \pm}(p)$ we look for the list of past passenger groups that take the $j$-th train in some phantom process $p$ :

$l_{j}=\left\{k: \exists p \leq j\right.$ such that $\Delta^{ \pm}(p)<0$ and $\left.V_{\theta}(j-1)<S(k)+\Delta^{ \pm}(p) \leq V_{\theta}(j)\right\}$,

which naturally may contain passenger groups in the past, for which $S(k)<$ $V_{\theta}(j-1)$. Because all these passenger groups satisfy $j=\kappa_{k}^{ \pm}(p)$, the corresponding contribution to the MVD estimator is:

$$
\sum_{k \in l_{j} p: V_{\theta}(j-1)<S(k)+\Delta^{ \pm}(p) \leq V_{\theta}(j)} T_{k}\left( \pm V^{ \pm}(j ; p)\right) \mathbf{1}_{\left\{\Delta^{ \pm}(p)>0\right\}} .
$$

In our computer program we have chosen to optimize the list updating structures in order to save both memory and computing time. A final correction must be considered: at the end of the day all passenger groups that arrived after the last train should be allowed to leave the metro. It may be necessary to generate the last trains for the dominated processes or force the last train to empty the station at the closing time $T$. On the other hand, a dominating process may have spanned the horizon $T$ before train $D_{\theta}(T)$ so a corresponding correction must be included in the program. Table 4 shows the results of the program with all the contribution of the phantom systems using the Maxwell/Weibull mixture distribution.

Comparing with the SF estimator, similar precision is obtained here 20 times faster. The improvement in variance reduction compared to the randomized

changed: $L_{(\mathrm{MVD})}$ estimation is not very dramatic, however, and we are currently studying methods for variance reduction that will make it possible to update the subway system as frequently as every month. 


\begin{tabular}{c|c}
$\mathrm{N}$ & $L_{(\mathrm{MVD})}$ \\
\hline 5000 & $58.722 \pm 5.9205$ \\
10000 & $58.277 \pm 4.1704$ \\
50000 & $56.671 \pm 1.8858$
\end{tabular}

Table 4: Averaged MVD Estimation

\subsection{Overview on Results}

Based on the numerical experiments, we summarize our results in Table 5.

Table 5: Overview on Numerical Experiments

\begin{tabular}{|c||c|c|c|c|}
\hline & Numerical Stability & Low Variance & Weak Conditions & Easy Implementation \\
\hline SPA & - & + & + & - \\
\hline SF & + & - & - & + \\
\hline MVD & + & + & - & - \\
\hline \hline
\end{tabular}

\section{Conclusion}

We have derived an SPA, SF and an MVD estimator for a problem in public transportation. For our model the SPA and MVD sensitivity estimators turn out to be intrinsically different and our analysis may serve as a counter-example to the folk belief that SPA and measure-valued differentiation yield in principle equivalent estimators. Efficient implementations of the SPA and MVD estimators suffer from the problem of biasedness, whereas the SF estimator can be implemented without difficulty in its unbiased form. When it comes to sample variance, the MVD estimator outperforms the others. In addition to that it turned out that the MVD analysis provided more structural insight.

\section{References}

[1] Brémaud, P., Vázquez-Abad, F. (1992) "On the pathwise computation of derivatives with respect to the rate of a point process: the phantom RPA method", Queueing Systems, Theory and Appl., 10: 249 - 270.

[2] M. Fu and J.-Q. Hu. Conditional Monte Carlo, Kluwer Academic, Boston, 1997.

[3] W.-B. Gong and Y.-C. Ho, Smoothed (conditional) perturbation analysis of discrete-event dynamical systems. IEEE Trans. Aut. Cont., vol. 39, no. 11, 1990, pp. 1218-1230.

[4] B. Heidergott and F. Vázquez-Abad, Measure-valued differentiation for stochastic processes: the finite horizon case. EURANDOM Report 2000-033, 2000 [This report is accessable via the web at http://staff.feweb.vu.tue.nl/bheidergott] 
[5] B. Heidergott and F. Vázquez-Abad, Measure-valued differentiation for stochastic processes: the random horizon case. Report GERAD G-2001-18, 2000 [This report is accessable via the web at http://staff.feweb.vu.tue.nl/bheidergott]

[6] B. Heidergott, G. Pflug and F. Vzquez-Abad. Measure-Valued Differentiation for stochastic systems: from simple distributions to Markov chains. (submitted) 2003 [This report is accessable via the web at http://staff.feweb.vu.tue.nl/bheidergott]

[7] G. Pflug. Optimisation of Stochastic Models. Kluwer Academic, Boston, 1996.

[8] M. Reiman and A. Weiss. Sensitivity Analysis for Simulations via Likelihood Ratios. Operations Research, 37: 830-844, 1989.

[9] S.M. Ross Applied Probability Models with Optimization Applications. Holden-Day, San Francisco, 1970.

[10] R. Rubinstein and A. Shapiro. Discrete Event Systems: Sensitivity Analysis and Optimization by the Score Function Method. Wiley, 1993.

[11] F.J. Vázquez-Abad (2000) "A Course on Sensitivity Analysis for Gradient Estimation of DES Performance Measures", Part I of III, in Discrete Event Systems, Analysis and Control, R. Boel and G. Stremersch Editors, Kluwer Academic Publishers, Boston: 3-28.

[12] F.J. Vázquez-Abad and B. Heidergott, "Gradient estimation for a problem in public transportation: A comparison of SPA, SF and MVD", invited paper, submitted to Conference on Decision and Control 2003.

[13] F. Vázquez-Abad and L. Zubieta, Distributed stochastic apporximations for adaptive frequency allocation in subway networks. Proceeding of the 39th Conference on Decision and Control, edited by IEEE Control Systems Scociety, 4729-4734, 2000.

[14] F. Vázquez-Abad and L. Zubieta, "Ghost Simulation Model for the Optimisation of an Urban Subway System", submitted.

[15] F. Vázquez-Abad and L. Zubieta. Simplifying sensitivity analysis for Intelligent Subway Control Proceedings of the Interational Industrial Simulation Conference 2003.

[16] W. Whitt, Continuity of Generalized Semi-Markov Processes Mathematics of Operations Research 5:494-501, 1980.

\section{Appendix}

\section{Sampling the Distributions}

For the MVD estiamtor it is necessary to generate variables from the Weibull $(2,1 / 2 \theta \sigma)$ and from a double-Maxwell $(\theta, \sigma \theta)$. The Weibull- $(\alpha, \beta)$-distribution is generated using the inverse function method (see [9]). The density is

$$
\mathbf{w}_{\alpha, \beta}(x)=\alpha \beta x^{\alpha-1} e^{-\beta x^{\alpha}},
$$


so, for $\alpha=2$ and $\beta=1 /(2 \theta \sigma)$, if $U$ is a uniform variate, then

$$
Z_{\theta}=\sqrt{-2 \theta \sigma \ln (1-U)}
$$

has the required distribution. For the double Maxwell distribution with parameters $(\mu, s)$, it is enough to devise an algorithm to generate a standard variable $X$ (zero mean and unit variance), because $s X+\mu$ has the required distribution. For the case $\mu=0, s=1$, we use first a composition argument: the distribution is symmetric around zero, so it is enough to be able to generate $X$ conditioned on $X \geq 0$, for which the density is:

$$
f_{0,1}^{\text {pos }}(x)=\frac{2}{\sqrt{2 \pi}} x^{2} e^{-x^{2} / 2}
$$

Generation of this random variable can now be performed with the acceptance/rejection test (see [9] for example), using a Weibull(2,1/4), whose density is $\mathbf{w}_{2,1 / 4}(y)=\frac{1}{2} y e^{-y^{2} / 4}$, so that the ratio of the densities is uniformly bounded:

$$
c=\max _{y \in \mathbb{R}}\left(f^{p o s}(y) / \mathbf{w}_{2,1 / 4}(y)\right)=4 / \sqrt{e \pi}=1.368793,
$$

which gives an acceptance probability of $73 \%$. The test function is:

$$
R(y)=\frac{f^{\text {pos }}(y)}{\mathbf{w}_{2,1 / 4}(y)} \frac{\sqrt{e \pi}}{4}=\sqrt{e / 2} y e^{-y^{2} / 4} .
$$

We generate the Weibull as we did in (17): let $U$ be a uniform variate, and set $Y=\sqrt{-4 \ln (1-U)}$. The $\mathrm{A} / \mathrm{R}$ test then can be simplified by evaluating the algebra: $R(Y)=\sqrt{e / 2} Y(1-U)$. The pseudo-code is as follows:

1. DO

$$
\begin{aligned}
& U_{1}=\text { Rand, set } Y=\sqrt{4 \ln \left(U_{1}\right)} \\
& U_{2}=\text { Rand, independent of } U_{1}
\end{aligned}
$$

WHILE $\left(U_{2}>\sqrt{e / 2} Y U_{1}\right)$

2. $U_{3}=$ Rand

3. If $U_{3}<0.5$ set $X=\mu+s Y$, otherwise $X=\mu-s Y$.

Figure 4 shows the Maxwell density against the empirical historgram using our code. To create the histogram we divided the interval $(-4.0,4.0)$ into 41 subintervals and pereformed $10^{5}$ calls to the generator.

\section{Derivation of MVD Formulas}

Let $\mathcal{N}_{\mu, s^{2}}$ be a Normal distribution with mean $\mu$ and variance $s$, with $s^{2}>0$. Denote the density of $\mathcal{N}_{\mu, s^{2}}$ by

$$
\phi_{\mu, s^{2}}=\frac{1}{s \sqrt{2 \pi}} e^{-\frac{1}{2}\left(\frac{x-\mu}{s}\right)^{2}}
$$




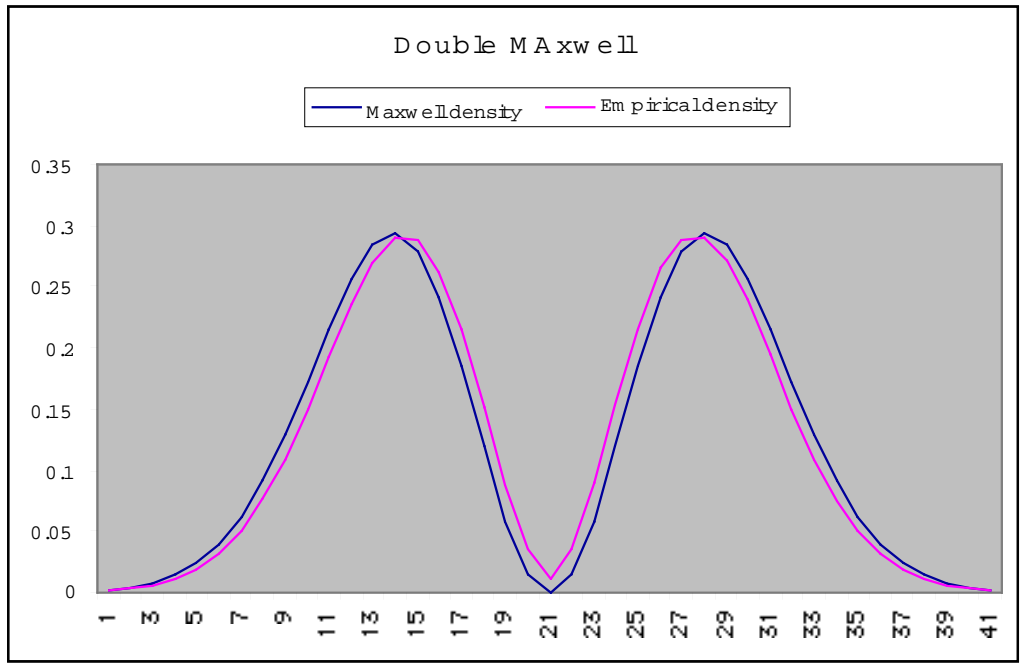

Figure 4: Generation of the double Maxwell $(0,1)$ distribution

Furthermore, denote by

$$
\mathbf{m}_{\mu, s^{2}}(x)=\frac{1}{s^{3} \sqrt{2 \pi}}(x-\mu)^{2} e^{-\frac{1}{2}\left(\frac{x-\mu}{s}\right)^{2}}
$$

the density of a double-sided Maxwell distribution with mean $\mu$ and shape parameter $s$, and denote the corresponding distribution by $\mathbf{M}_{\mu, s^{2}}$. Moreover, recall that

$$
\mathbf{w}_{\alpha, \beta}(x)=\alpha \beta x^{\alpha-1} e^{-\beta x^{\alpha}}
$$

denotes the density of a Weibull distribution with parameter $\alpha$ and $\beta$, and denote the distribution function by $\mathbf{W}_{\alpha, \beta}$. If $Y$ is distributed according to Weibull- $(\alpha, \beta)$-distribution, then we denote the distribution of the random variable $Y+\delta$ by $\mathbf{W}_{\alpha, \beta}^{(+, \delta)}$ and that of the random variable $\delta-Y$ by $\mathbf{W}_{\alpha, \beta}^{(-, \delta)}$. The corresponding densities are denoted by $\mathbf{w}_{\alpha, \beta}^{(+, \delta)}$ and $\mathbf{w}_{\alpha, \beta}^{(-, \delta)}$, respectively. It is well known that

$$
\frac{d}{d \mu} \phi_{\mu, s^{2}}(x)=\frac{1}{s \sqrt{2 \pi}}\left(\mathbf{w}_{2,\left(2 s^{2}\right)^{-1}}^{(+, \mu)}(x)-\mathbf{w}_{2,\left(2 s^{2}\right)^{-1}}^{(-, \mu)}(x)\right)
$$

and

$$
\frac{d}{d s} \phi_{\mu, s^{2}}(x)=\frac{1}{s}\left(\mathbf{m}_{\mu, s^{2}}(x)-\phi_{\mu, s^{2}}(x)\right)
$$

see $[7,6]$. Applying the chain rule of calculus therefore yields

$\frac{d}{d \theta} \phi_{\theta,(\theta \sigma)^{2}}(x)=\frac{1}{\theta \sigma \sqrt{2 \pi}}\left(\mathbf{w}_{2,\left(2 \theta^{2} \sigma^{2}\right)^{-1}}^{(+, \theta)}(x)-\mathbf{w}_{2,\left(2 \theta^{2} \sigma^{2}\right)^{-1}}^{(-, \theta)}(x)\right)+\sigma \frac{1}{\theta \sigma}\left(\mathbf{m}_{\theta,(\theta \sigma)^{2}}(x)-\phi_{\theta,(\theta \sigma)^{2}}(x)\right)$.

Let

$$
c_{\theta}=\frac{1}{\theta \sigma \sqrt{2 \pi}}+\frac{1}{\theta}=\frac{1+\sigma \sqrt{2 \pi}}{\theta \sigma \sqrt{2 \pi}}
$$


and set

$$
\begin{gathered}
p^{\mu}=\frac{1}{c_{\theta}} \frac{1}{\theta \sigma \sqrt{2 \pi}}=\frac{1}{1+\sigma \sqrt{2 \pi}} \\
p^{s}=\frac{1}{c_{\theta} \theta}=\frac{\sigma \sqrt{2 \pi}}{1+\sigma \sqrt{2 \pi}},
\end{gathered}
$$

then

$\frac{d}{d \theta} \phi_{\theta,(\theta \sigma)^{2}}(x)=c_{\theta}\left(p^{\mu}\left(\mathbf{w}_{2,\left(2 \theta^{2} \sigma^{2}\right)^{-1}}^{(+, \theta)}(x)-\mathbf{w}_{2,\left(\theta^{2} \sigma^{2}\right)^{-1}}^{(-, \theta)}(x)+p^{s}\left(\mathbf{m}_{\theta,(\theta \sigma)^{2}}(x)-\phi_{\theta,(\theta \sigma)^{2}}(x)\right)\right)\right.$.

In words, the derivative of the distribution $\mathcal{N}_{\theta,(\theta \sigma)^{2}}$ with respect to $\theta$ is the sum of the derivative of $\mathcal{N}_{\theta,(\theta \sigma)^{2}}$ with respect to the mean and the derivative of $\mathcal{N}_{\theta,(\theta \sigma)^{2}}$ with respect to the standard deviation. Rearranging terms yields

$$
\frac{d}{d \theta} \phi_{\theta,(\theta \sigma)^{2}}(x)=c_{\theta}\left(\phi_{\theta,(\theta \sigma)^{2}}^{+}(x)-\phi_{\theta,(\theta \sigma)^{2}}^{-}(x)\right),
$$

where

$$
\phi_{\theta}^{+}(x):=\phi_{\theta,(\theta \sigma)^{2}}^{+}(x)=p^{\mu} \mathbf{w}_{2,\left(2 \theta^{2} \sigma^{2}\right)^{-1}}^{(+, \theta)}(x)+p^{s} \mathbf{m}_{\theta,(\theta \sigma)^{2}}(x)
$$

and

$$
\phi_{\theta}^{-}(x):=\phi_{\theta,(\theta \sigma)^{2}}^{-}(x)=p^{\mu} \mathbf{w}_{2,\left(2 \theta^{2} \sigma^{2}\right)^{-1}}^{(-, \theta)}(x)+p^{s} \phi_{\theta,(\theta \sigma)^{2}}(x) .
$$

The above result allows for the following interpretation: we may interpret the derivative as the mixture of the partial derivatives with respect to the mean and the standard deviation, respectively. 